\title{
DOS CONCEITOS DE MORTE AOS CRITÉRIOS PARA O DIAGNÓSTICO DE MORTE ENCEFÁLICA
}

\author{
VENÂNCIO PEREIRA DANTAS FILHO*, LUIS ANTONIO DA COSTA SARDINHA**, \\ ANTONIO LUIS EIRAS FALCÃO**, SEBASTIĀO ARAĹUO***, \\ RENATO GIUSEPPE GIOVANNI TERZI ${ }^{* * *}$, BENITO PEREIRA DAMASCENO*****
}

\begin{abstract}
RESUMO - Os autores apresentam considerações a respeito dos conceitos de morte e morte encefálica, bem como dos aspectos legais para o seu diagnóstico no Brasil. Apresentam também o Protocolo para Diagnóstico de Morte Encefálica atualmente em vigor no Hospital das Clínicas da Universidade Estadual de Campinas, revisto e atualizado conforme a lei vigente no país, com a padronização técnica dos testes diagnósticos, ressaltando a importância de um posicionamento ético maduro frente a essa desafiante e cada vez mais frequente situação.
\end{abstract}

PALAVRAS-CHAVE: morte encefálica, diagnóstico, protocolo, transplante de órgãos, lei.

Death concepts and brain death diagnostic criteria

ABSTRACT - The authors present considerations about death and brain death concepts, as well the legal aspects for its diagnosis in Brazil. They also present the UNICAMP Protocol for the Diagnosis of Brain Death, revised and according with the current law, with standard techniques for the diagnostic exam. They emphasize the importance of a mature ethical position for this frequent and challenging situation.

KEY WORDS: brain death, diagnosis, protocol, transplantation, law

No mundo ocidental, o conceito tradicional de morte, que se encontra profundamente enraizado em cada um de nós, baseia-se principalmente em duas fortes influências históricas culturais: a influência da filosofia grega da antiguidade, que estabelece o coraçāo como órgão sede da alma e, portanto, de todas as emoçōes humanas (como a coragem e o amor) e a influência religiosa judaico-cristã, muito forte durante a Idade Média, que estabelece a respiráção como processo vital fundamental, uma vez que "Deus criou o homem do barro da terra e soprou-lhe pelas narinas o Sopro da Vida, e o homem tornou-se um ser vivente" $(\mathrm{Gen} 2,7)^{2.10}$. 0 proprio Código Civil Brasileiro (Art. $4^{0} \mathrm{CC}$ ) define pessoa natural como todo aquele "nascido de mulher" com vida. Considera-se a respiraçăo como sendo a melhor prova do nascimento com vida (daí a importância médicolegal das docimásias respiratórias)".

Os critérios médicos tradicionais para o diagnóstico de morte foram também influenciados por esses conceitos, ressaltando a ausência de função cardiocirculatória e respiratória como premissa básica para esse diagnóstico. Cabe aqui diferenciar o termo critério (norma objetiva que serve para o julgamento) do termo conceito (idéia, opinião, muito mais subjetiva, a respeito de um determinado assunto).

O diagnóstico tradicional de morte baseia-se nos sinais abióticos (ou tanatognósticos). $O$ estudo sistematizado desses sinais classifica-os como: imediatos, aqueles que se seguem imediatamente após a morte (entre outros a imobilidade, a ausência da consciência, parada cardiocirculatória e respiratória, relaxamento dos esfincteres, inclusive a midríase, entre outros); consecutivos, que se seguem apos horas ou dias (manchas hipostáticas, mancha verde abdominal, hipotermia, rigidez, entre outros); e os sinais abióticos tardios, que aparecem dias ou semanas após a morte (sinais transformativos do cadáver, como a putrefaçāo). $O$ estudo da cronologia do aparecimento dos sinais abióticos (cronotanatognose) tem importância médico-legal relevante e levou ao

Hospital das Clínicas da Universidade Estadual de Campinas (HC/UNICAMP): *Médico Neurocirurgiāo, Disciplina de Neurocirurgia; **Médico Neurologista, Unidade de Terapia Intensiva; ***Diretor Técnico, Unidade de Terapia Intensiva; ****Coordenador do Núcleo de Assistência Médica Intensiva; *****Chefe do Departamento de Neurologia e Neurocirurgia da Faculdade de Ciências Médicas da UNICAMP. Aceite: 31-julho-1996. 
estabelecimento dos sinais tanatognósticos especiais, que compreendem uma série de técnicas especiais para a constatação da morte muito recente (uso do eletrocardiograma, cardiopuntura, arteriotomia - entre muitas outras)

A análise da sistematização tradicional dos sinais abióticos nos leva a três importantes conclusões: primeiramente, notamos que nāo há sinal patognomônico de morte (pelo menos morte recente); em segundo lugar fica claro que há muito se sabia que a morte não é evento único e sim uma sequência de eventos; e, por último, esse diagnóstico é tanto mais difícil quanto mais recente é a morte, o que sempre causou insegurança e medo do diagnóstico de morte real em um estado de morte apenas aparente. $O$ próprio Código do Processo Penal Brasileiro só autoriza a realização de autópsias após 6 horas da constatação da morte para diminuir o risco desse tipo de erro (Art. 162 CPP).

Podemos concluir, portanto, que a morte sempre foi tradicionalmente entendida como uma sequência inexorável de eventos, que văo progressivamente se instalando, tornando cada vez mais claro seu diagnóstico ${ }^{10}$.

\section{MUDANÇA DO PARADIGMA}

Com o recente desenvolvimento de técnicas de ressuscitação cardiorrespiratória e de suporte avançado de vida foi se tornando possível a reversão de número cada vez maior de "paradas cardíacas e respiratórias", levando à reintegração de grande número de indivíduos à sociedade mesmo após a ocorrência desses eventos.

Por outro lado, num contingente também cada vez maior de indivíduos, a reanimaçăo cardiorrespiratória só foi possível após a instalação de extensas e irreversíveis lesões do sistema nervoso central (SNC), passando assim, os outros sistemas reanimados a funcionarem artificialmente mantidos e independentes entre si, uma vez cessada a açāo integradora do SNC irreversivelmente lesado. Vale lembrar que o tecido nervoso, por suas peculiaridades metabólicas, altas demandas e mínimas reservas de oxigênio e glicose, é particularmente mais vulnerável à privação desses substratos decorrente das situaçōes de parada cardiorrespiratória que qualquer outro tecido do organismo. Podemos até mesmo inferir que a "morte" do SNC seja o primeiro evento da sequência de eventos já citada, uma vez que a ausência da açăo integradora do SNC leva inexoravelmente à falência progressiva dos demais sistemas, independente da qualidade dos métodos de suporte artificial ${ }^{10}$.

Assim, o conceito de "morte de todo o corpo" foi sendo substituido pelo conceito de "morte do corpo como um todo" e os tradicionais critérios cardiorrespiratórios foram dando lugar aos critérios neurológicos para o diagnóstico de morte (já que o SNC é o responsável pelo funcionamento dos demais sistemas do corpo como um todo) ${ }^{10}$.

\section{DEFINIÇÃO DO CONCEITO MORTE}

Apesar de todo avanço científico da medicina atual, a exata definição do conceito de morte ainda aparece como questão desafiadora, já que não contamos ainda com uma exata definição da sua situação oposta e excludente: a vida".

No Brasil, a lei federal 8489 de 1992, regulamentada pelo decreto 879 de 1993, cita morte encefálica como "a morte definida como tal, pelo Conselho Federal de Medicina e atestada por médico" (Art. 32, V), seguindo os critérios da Resolução 1346 de 1991 do Conselho Federal de Medicina. Mesmo com essas disposiçðes legais, 0 assunto em nosso meio está longe de consenso satisfatório ${ }^{8}$.

O conceito atual de morte encefálica (ME): "Ausência total e irreversivel das funçðes do SNC" tem sido campo de muita polêmica científica, o que dificulta sobremaneira a definição de critérios uniformes para o seu diagnóstico. Questōes conceituais sobre a totalidade e a irreversibilidade das lesōes do SNC têm sido recentemente levantadas, bem como a introdução de novos métodos diagnósticos que possam certificar melhor e mais rapidamente a $\mathrm{ME}$.

A necessidade de posicionamento ético e legal frente à racionalização da ocupação de leitos hospitalares, bem como a identificação precoce de potenciais doadores de órgãos e tecidos humanos são fatores que acaloram ainda mais essa polêmica?.

Discute-se hoje a equivalência entre os conceitos de "morte de todo o encéfalo" e "morte do encéfalo como um todo" ${ }^{\circ}$, chegando-se a discussð̄es conceituais muito mais amplas como as situaçōes de morte iminente (anencéfalos ou condenados à pena de morte prestes a serem executados) poderem ou năo ser considerados doadores de órgãos ainda em vida.

\section{ASPECTOS TÉCNICOS DO DIAGNÓSTICO DE MORTE ENCEFÁLICA}

O diagnóstico de ME no Brasil é atualmente baseado na Resolução 1346 de 1991 do CFM. Essa resolução orienta inclusive a elaboração de protocolos, aprovados pelas Comissð̌es de Ética de cada instituição, para esse fim.

No Hospital das Clínicas da Universidade Estadual de Campinas já contávamos com um protocolo desde o ano de $1990^{3}$, o qual, após a publicaçāo da Resolução do CFM, foi atualizado por uma Comissão Multidisciplinar, constituída de docentes do referido hospital, sob a coordenação de um dos autores (BPD). 


\section{PROTOCOLO PARA DIAGNÓSTICO DE MORTE ENCEFÁLICA HC/UNICAMP}

NOME: IDADE: HC:

DATA DA INTERNAÇĀO: HORA:

EXAMES CLÍNICOS - DATA E HORA:

$1^{2}$ EXAME: h. 29 EXAME: h.

\section{HISTÓRIA PROGRESSIVA E DIAGNÓSTICO DO COMA:}

\section{CRITÉRIOS DE INCLUSĀO E EXCLUSĀO:}

a - Causa imediata do coma:

b - Anormalidades corrigiveis (Presentes ou Ausentes):

c - Hipotermia - temperatura axilar - $\quad 1^{2}$ EXAME: $2^{2}$ EXAME:

d - Ausência do uso de drogas ou confirmar o tempo mínimo de suspensão:

Barbitúricos: Opiáceos:

e - Coma arresponsivo (Sim ou Não): $1^{2}$ EXAME: , Diazepínicos e Relaxantes musculares:

3. REFLEXOS PARA AVALIAÇÃO DA FUNÇÃO DO TRONCO CEREBRAL: (Presentes ou Ausentes): 19 EXAME $2^{2}$ EXAME

a - Fotomotor direto:

b - Cómeo-palpebral:

c - Reflexos orofaríngeos (vômito):

d - Reflexos traqueais (tosse):

e - Óculo-cefálico:

f - Óculo-vestibular:

$\mathrm{g}$ - À estimulação dolorosa intensa supra-orbital observa-se:

Reação pupilar (R. Cílio-espinal):

Alteraçāo da frequência cardíaca:

Reações motoras:

4. TESTE DE APNÉIA:

Movimentos Respiratórios (Presentes ou Ausentes) $1^{\text {Q EXAME: }}$ $\left(\mathrm{PaCO}_{2}=\right.$ mmHg) $2^{2} \mathrm{EXAME}$ $\left(\mathrm{PaCO}_{2}=\right.$ $\mathrm{mmHg}$ )

5. ANGIOGRAFIA CEREBRAL RADIOISOTÓPICA:

6. OUTROS EXAMES COMPLEMENTARES (quando realizados):

CONCLUSÃO: Considerando os achados acima, certificamos o diagnóstico de :

MEDICOS:

NOME:

ASS.:

CRM:

NOME: ASS: CRM: 


\section{INSTRUCÓES PARA APLICAÇOO DO PROTOCOLO DE MORTE ENCEFALICA}

Este Protocolo obedece os critérios estabelecidos pela Resolução 1346/91 do Conselho Federal de Medicina.O diagnóstico de morte encefálica deve basear-se (1) na ausência de funçöes cerebrais, incluindo aquelas do tronco cerebral (podendo, porém, estar presentes funções mediadas pela medula espinhal) e (2) na irreversibilidade deste estado pelo menos 6 horas após sua constatação inicial.

1. COMPETÊNCIA: O Protocolo deve ser aplicado e assinado por dois médicos com adequado treinamento e experiência na aplicação do mesmo (sendo pelo menos um deles neurologista ou neurocirurgião), mediante dois exames clínicos (estritamente conforme as instruções abaixo), com intervalo mínimo de 6 horas, e com confirmação dos Critérios de Inclusão e Exclusảo antes de cada exame. Nāo deve haver participação de médicos da equipe de Transplantes de Órgãos no processo de diagnóstico de morte encefálica.

2. CRITÉRIOS DE INCLUSÃO E EXCLUSÃO: Devem ser confirmados antes de cada exame.

Idade: Săo excluídos deste Protocolo pacientes com menos de 2 anos de idade.

Causa imediata do coma: $O$ seu conhecimento é obrigatório.

Anormalidades corrigiveis: Devem ser excluídas antes de cada exame (por exemplo hipotensāo, hipoglicemia. alteraçðes hidroeletrolíticas, etc).

Hipotermia: O paciente deve ser aquecido a pelo menos $35^{\circ} \mathrm{C}$ para a realização dos exames.

Ausência do uso de drogas: Excluir intoxicaçōes por drogas depressoras do sistema nervoso central. Na suspeita ou confirmação de intoxicação precedendo a avaliação, aguardar o tempo mínimo de metabolização e excreção, ou seja, 24 horas para barbituratos, 12 horas para opiáceos e 8 horas para diazepínicos e relaxantes musculares.

Coma arresponsivo: Ausência de atividade motora espontânea ou à estímulos dolorosos eficientes, aplicados em regiōes inervadas por nervos cranianos (incluindo ausência de reaçōes de decorticaçăo, decerebração e tremores). Movimentos mediados pela medula espinhal podem, eventualmente, estar presentes.

\section{REFLEXOS PARA AVALIAÇĀO DA FUNÇĀO DO TRONCO CEREBRAL:}

Eotomoter: Não deve haver qualquer reação pupilar após pelo menos 10 segundos (para afastar a possibilidade de pupilotonia) de estimulação da pupila com oftalmoscópio de luz forte (midriase não é condição indispensável).

Cómeo-palpebral: Não deve haver reaçăo de fechamento ocular após estimulaçăo eficiente e cuidadosa de ambas as cómeas (prevenir lesð̄es corneanas pela possibilidade de doação).

Reflexos orofarfngeos ou orotraqueais: Não deve haver reflexo faríngeo ou de vômito à estimulação da mucosa orofaríngea, nem reflexo de tosse à estimulação da mucosa traqueal.

Reflexe oculo-cefálico: Não deve haver movimentos dos olhos (conjugados ou não) à rotação passiva da cabeça para ambos os lados, nem à flexão e extensão da cabeça.

Reflexo óculo-yestibular: Não deve haver movimentos dos olhos (conjugados ou não) à irrigação de cada conduto auditivo externo com água ou soro fisiologico à $0^{\circ} \mathrm{C}$ por pelo menos 1 minuto (com 5 minutos de intervalo entre a irrigaçāo de um conduto e outro), estando o paciente em proclive de $30^{\circ}$. Certificar-se, mediante otoscopia, que os condutos auditivos estão pérveos $e$ as membranas timpânicas integras (perfuraçð̄es contra-indicam o exame).

À estimulacão dolorosa intensa da regiāo supra-orbital (forte compressão com o dedo), mantida por pelo menos 10 segundos, devem estar ausentes: (1) reações pupilares (dilatação mediada pelo reflexo cillio-espinhal); (2) alteraç̋es sienificativas da frequência cardíaca, observadas no osciloscópio do monitor cardíaco (levar em consideraçāo as pequenas variaçðes da frequência cardíaca que podem ocorrer antes da estimulaçăo); e (3) reaç̃es motoras da face. tronce ou membros.

4. TESTE DE APNÉLL: Após 20 minutos de ventilação com $\mathrm{O}_{2}$ a $100 \%$, manter 15 minutos de apnéia com catéter de $\mathrm{O}_{2}$ na cânula traqueal a 6 litros por minuto (durante todo o tempo de apnéia). Não deve haver movimentos respiratórios durante a realização do exame. Colher gasometria arterial no início e no final do período de apnéia para quantificar os níveis de $\mathrm{O}_{2}$ e $\mathrm{CO}_{2}\left(\mathrm{PaO}_{2}\right.$ mínima para iniciar a apnéia $=200 \mathrm{mmHg}$ e $\mathrm{PaCO}_{2}$ mínima no final da apnéia $=60 \mathrm{mmHg}$ ).

5. ANGIOGRAFIA CEREBRAL RADIOISOTÓPICA ou angiografia convencional devem mostrar ausência de perfusão encefálica.

6. OUTROS EXAMES COMPLEMENTARES: Devem mostrar a ausência de perfusão, atividade metabólica ou bioelétrica do encéfalo. O eletroencefalograma (EEG) deve ser considerado isoelétrico (ausência de variaçðes dos potenciais elétricos acima de 02 microvolts durante dois registros de 30 minutos, com intervalo de pelo menos 6 horas entre um e outro). Anote o resultado de outros exames que tragam informações sobre a função ou estrutura do encéfalo (p.ex. tomografia computadorizada de crânio, ressonância magnética do encéfalo, etc).

7. APÓS O DIAGNÓSTICO DE MORTE ENCEFÁLICA, preenchimento do Protocolo e assinatura do termo, os médicos devem imediatamente comunicar o fato aos responsáveis legais do paciente para a autorização da doação de ớgāos (ou para o desligamento do aparelho de ventilação artificial, caso o paciente não seja um potencial doador). Uma vez autorizada a doação, os médicos devern imediatamente comunicar a equipe de Captação de Órgãos da Instituiçāo.

8. A APLicaÇÃo do protocolo E O diagnóstico de morte encefálica Nāo NECESSTTAM DA AUTORIZAÇĀO DOS RESPONSÁVEIS LEGAIS DO PACIENTE. 
Esse protocolo visa a uniformizaçăo dos procedimentos técnicos para o diagnóstico de ME (Quadros ) e 2, frente e verso da folha única do Protocolo).

\section{ASPECTOS GERAIS}

Competência. O diagnóstico deve ser firmado, pelo menos por dois médicos, de preferência o médico assistente do doente, que tem conhecimento da causa imediata do coma, e um outro, da equipe de neurologia ou neurocirurgia, que deverá auxiliar aa aplicaçlo e interpretaç⿸厃㔾̉o dos testes diagnósticos. Não deve haver qualquer participaçăo dos membros da equipe de transplantes no processo de diagnóstico de ME.

Critérios de inclusda e exclusāo. Só devem ser submetidos aos testes diagnósticos os pacientes que se enquadrem nos seguintes critérios:

1. Idade. Somente pacientes com dois anos ou mais. Crianças abaixo desta idade devem ser avaliadas segundo outros criterios, visto a dificuldade de aplicaçăo e interpretaçāo dos testes, bem como a variabilidade de suas respostas.

2. Causa imediata do coma. É imprescindível o seu conhecimento para nos certificarmos da fidedignidade das respostas aos testes, afastando assim, outras causas de depressăo da funçăo do SNC.

3. Anormalidades corrigiveis. Devem ser definitivamente exclufdas e compensadas (por exemplo hipotensão arterial, alteraçסes hidroeletrolf́ticas e hipoglicemia).

4. Hiposermia. A chamada "hipotermia acidental" năo constitui problema frequente em paises de clima quente, porém deve ser sempre excluída. A hipotermia terapêutica ou por lesão do SNC, quando presente, merece cuidadosa interpretaçăo por parte dos médicos responsáveis pelo diagnóstico de ME. Em caso de dúvidas devemos aquecer o doente até temperatura mínima de $35^{\circ} \mathrm{C}$ por ocasiáo do exame.

5. Drogas depressoras do SNC. E obrigatória a exclusão de intoxicações por essas drogas. No caso do seu uso terapéutico precedendo o diagnóstico de $\mathrm{ME}$, devemos aguardar um tempo minimo de metabolizaçăo e excreçæ̃. após a total suspenstio do seu uso (24 horas para barbitúricos, 12 horas para opiáceos $\mathrm{e} 8$ horas para diazepínicos e relaxantes musculares). Sempre que possivel, as intoxicaçoes por drogas devem ser afastadas atraves da respectiva dosagem sanguínea.

6. Coma arresponsivo. Nlo deve haver qualquer tipo de resposta motora, inclusive respostas inapropriadas em decorticaçăo e decerebraçăo ou tremores após estimulaçăo dolorosa eficiente aplicada em regiócs inervadas por nervos cranianos. Em algumas situaçס̄es de ME constatada, podem aparecer movimentos de vários tipos c amplitudes, espontâneos ou provocados, as vezes relacionados ao teste de apnetia ou a perfodos de hipoxia. 0 reconhecimento e a interpretacllo cuidadosa desses "movimentos medulares persistentes" cabem aos médicos que aplicam os testes, devendo, eventualmente, ser cuidadosamente considerados junto a colegas e pessoal de enfermagem menos avisados ${ }^{5,11,14}$.

\section{EXAMES ESPECFFICOS PARA O DIAGNOSTICO DE ME}

Devem ser realizados dois exames completos, corn intervalo mínimo de 6 horas, sendo que os criterios de inclusăo e exclusăo devem ser confirmados antes de cada exame.

Reflexos para avaliacäo da funfäo do tronco cerebral Devem estar ausentes nos dois exames realizados sob padronizaço técnica.

Teste de apnéia. É de fundamental importância para o diagnóstico de ME. Deve ser realizado após cada exame clínico, com controle gasométrico no início e final do teste. Pacientes com a $\mathrm{PaO}_{2}$ abaixo de $200 \mathrm{~mm} \mathrm{Hg}$, após 20 minutos de ventilaçăo com $\mathrm{O}_{2}$ a 100\%, năo devem ser submetidos ao teste pelo risco de descompensaçăo hipoxica durante ele. Os niveis de $\mathrm{PaCO}_{2}$, apos 15 minutos. de apneia com catéter de $\mathrm{O}_{2}$ na chinula traqueal a 6 litros por minuto, devem ser quantificados para nos certificarmos que ultrapassaram os limites de excitaçżo dos centros respiratórios do tronco cerebral, confirmando a leş̄o deles. Apesar de algumas divergencias quanto aos níveis da $\mathrm{PaCO}_{2}^{1,9,12}$, consideramos o nível de $60 \mathrm{~mm} \mathrm{Hg}$ como o mínimo satisfatório ${ }^{13}$. Sao descritos movimentos toracicos, mediados a nivel medular, durante o teste de apneia, em pacientes com ME confirmada ("spinal respiratory-like movements") ${ }^{12}$. Devemos, portanto, estar preparados para o reconhecimento c a correta interpretaçăo desses "movimentos medulares persistentes" durante a aplicaçăo do teste de apneia. Observaqbes pessoais sugerem que o uso do capnógrafo durante o teste, demonstrando a nâo existencia de $\mathrm{CO}_{2}$ nas vias aéreas, auxilia a confirmaçáo da ausencia de movimentos respiratórios.

Exames complementares. Apesar do diagnóstico de ME ser eminentemente clinico, este deve ser respaldado legalmente, pelo menos por um exame grafico subsidiário para sua documentaç̋o (principalmente em pacientes candidatos a doaçăo de órglos). A angiografia cerebral convencional (por punçio direta ou por cateterismo) ou radioisotópica prestam-se para demonstrar a auséncia de fluxo sanguineo cerebral. Recentemente, o uso do Doppler transcraniano tem-se mostrado util para esse mesmo fim, embora menos seguro que a angiografia devido aos falsos resultados negativos (ate 10\%)4. O eletrencefalograma (EEG), nto demonstrando alteraçós 
dos potenciais elétricos acima de 2 microvolts durante 30 minutos, em pelo menos dois registros com intervalo mínimo de 6 horas, confirma a ausência de atividade elétrica encefálica, o mesmo sendo demonstrado pela inexistência de ondas II, III, IV e V nos potenciais evocados do tronco cerebral. Diferenças artério-jugulares mínimas do conteúdo de $\mathrm{O}_{2}$ demonstram ausência de extração cerebral de $\mathrm{O}_{2}$, indicando a inexistência de atividade metabólica cerebral. As angiografias cerebrais e o EEG são exames subsidiários mais tradicionais e de maior confiabilidade para o diagnóstico de ME, podendo ser utilizados isoladamente, enquanto os demais exames, ainda de aceitação controversa, são considerados opcionais neste protocolo.

\title{
RECOMENDAÇÕES ADICIONAIS
}

$O$ diagnóstico de $\mathrm{ME}$ deve ser firmado sob rígido protocolo, por médicos cientes das responsabilidades éticas e legais que ele acarreta.

Trata-se de diagnóstico cvolutivo, necessitando de no mínimo 6 horas para ser firmado. Devemos evitar diagnósticos "instantâneos", que "rotulem" o paciente, bem como apressar a evolução da investigação. Os critérios de inclusão e exclusão devem ser fielmente considerados antes de cada exame clínico, pois vale lembrar que lidamos com pacientes gravíssimos e potencialmente recuperáveis até que se prove o contrário, pela confirmaçåo da $\mathrm{ME}^{3}$.

Após a constatação da ME, o protocolo deve ser preenchido e assinado pelos dois médicos responsáveis pelo diagnóstico, sendo compulsória a notificação da central de captação de órgãos.

\section{POSICIONAMENTO dO MÉDICO FRENTE O ASSUNTO}

\author{
"A ciência fez de nós deuses antes mesmo de merecermos ser homens." \\ Jean Rostand (1894-1977) - Biologo e escritor francês
}

O médico atual, formado para exercer uma medicina cada vez mais sofisticada, com exames, procedimentos cirúrgicos e fármacos cada vez mais complexos e caros, muitas vezes pressionado por longas jornadas de trabalho e plantões estafantes, acaba por criar mecanismos psicológicos de defesa contra a morte, a qual representa a frustração total de todas as suas expectativas e esforços (além de lhe trazer sempre a consciência da própria morte, uma vez que somente o tempo o separa da situação do paciente moribundo à sua frente).

Por outro lado, a sociedade atual tem exigido do médico, de forma cada vez mais intensa, um posicionamento ético e legal imediato a respeito de todos os assuntos, principalmente os relacionados com a morte. Vale lembrar também que esta nossa sociedade pluralista atual encontra-se totalmente à mercê de formadores de opinião de intençōes nem sempre retas.

Cabe, portanto, ao médico, primeiramente a reflexão e o amadurecimento dos seus próprios conceitos pessoais a respeito da morte, para um adequado posicionamento individual e posteriormente coletivo a respeito do assunto morte encefálica. É também imprescindível o estudo e a atualização constantes dos conceitos e critérios relacionados com o seu diagnóstico.

Somente dessa forma estará o médico bem preparado para enfrentar esta conflituosa e desafiante situação; cada vez mais frequente em sua prática diária.

\section{REFERÊNCIAS}

1. Benzel EC, Gross CD, Hadden TA, Kesterson L, Landreneau MD. The apnea test for the determination of brain death. J Neurosurg 1989;71:191-194.

2. Cowley LT, Young E, Raffin TA. Care of dying: an ethical and historical perspective. Crit Care Med 1992;20:1473-1482.

3. Dantas VP Filho, Torres MP, Araújo S, Vieira RJ, Falcão ALE, Sardinha LAC, Terzi RGG. Morte encefálica: consideraçōes gerais e protocolo para o seu diagnóstico. Rev Bras Terap Intens 1992;4:50-54.

4. Determining brain death in adults. Report of the Quality Standards Subcommittee of the AAN. In: World Neurology, 1995.

5. Heytens L, Verlooy J, Gheuens J, Bossaert L. Lazarus sign and extensor posturing in a brain-dead patient: case report. J Neurosurg 1989;71:449-451.

6. França GV. Medicina legal.Ed3. Rio de Janeiro: Guanabara Koogan, 1991:238-286.

7. Fuhrer MCA. Resumo de direito civil. Ed7. São Paulo: Editora Revista dos Tribunais, 1990:12-30.

8. Gomes JCM. Ética médica e UTI: reflexōes à luz do Código de Ética. J Bras Med 1992;62:48-57.

9. Manreza LA, Stávale MA. Consideraçớes básicas sobre morte encefálica. In Stávale MA (ed). Bases da terapia intensiva neurológica. São Paulo: Livraria Santos, 1996:647-652.

10. Pallis C. Brainstem death. In Vinken PJ, Bruyn GW, Klawans HL (eds) and Braakman R (co-ed). Handbook of Clinical Neurology, Vol 13(57): Head injury. Amsterdam: Elservier, 1990:441-496.

11. Ropper AH. Unusual spontaneous movements in brain-dead patients. Neurology 1984;34:1089-1092.

12. Ropper AH, Kennedy SK, Russel L. Apnea testing in the diagnosis of brain death. J Neurosurg 1981;55:942-946.

13. Schafer JA, Caronna JJ. Duration of apnea needed to confirm brain death. Neurology 1978;28:661-666.

14. Turmel A, Roux A, Bojanowski MW. Spinal man after declaration of brain death. Neurosurgery $1991 ; 28: 298-302$. 